\title{
Chiral Oxazaborolidine-Aluminum Bromide Complexes Are Unusually \\ Powerful and Effective Catalysts for Enantioselective Diels-Alder Reactions
}

\author{
Duan Liu, Eda Canales and E. J. Corey* \\ Department of Chemistry and Chemical Biology \\ Harvard University, 12 Oxford Street, Cambridge, Massachusetts 02138, USA
}

\section{Supporting Information}

Materials and Methods. Unless stated otherwise, reactions were performed in flame-dried glassware under a positive pressure of nitrogen using freshly distilled dry solvents. Thin-layer chromatography (TLC) was performed using E. Merck silica gel $60 \mathrm{~F}_{254}$ precoated plates $(0.25$ $\mathrm{mm})$. Flash chromatography was performed using Baker silica gel (40 $\mu \mathrm{m}$ particle size). NMR spectra were recorded on Varian Innova-500, or Mercury-400 instruments and calibrated using residual undeuterated solvent as an internal reference. IR spectra were recorded on Avatar 360 FT-IR spectrometer. Low-resolution and high-resolution mass spectral analyses were performed at the Harvard University Mass Spectrometry Center. Analytical high performance liquid chromatography (HPLC) was performed on Isco 2350 Series or Waters 626 HPLC using the indicated chiral column. Gas chromatography (GC) analyses were performed on HewlettPackard 6850 Series GC System equipped with flame ionization detector using a J \& W Scientific Cyclosil-B column (30 m x $0.25 \mathrm{~mm})$ or a $\gamma$-TA column $(30 \mathrm{~m} \times 0.25 \mathrm{~mm})$. Commercial grade reagents and solvents were used without further purification except as indicated below. Dichloromethane was distilled from calcium hydride. Cyclopentadiene was 
generated from the dimer at $220^{\circ} \mathrm{C}$ and stored under nitrogen at $-80{ }^{\circ} \mathrm{C}$. Diethyl fumarate, cyclopentenone, cyclohexenone, furan, 2,5-dimethylfuran, 1,3-cyclohexadiene, and 2,3dimethylbutadiene were distilled from $\mathrm{CaH}_{2}$ and stored under nitrogen at $-20{ }^{\circ} \mathrm{C}$. 1,4Napthoquinone (obtained from Aldrich in 97\% purity) was dried by azeotrope distillation (refluxing toluene, Dean-Stark), upon cooling crystalline 1,4-napthoquinone was obtained.

Oxazaborolidine (S)-1. A 100-mL, two-necked, round-bottomed flask equipped with a stir bar, a glass stopper and a 50-mL pressure-equalizing addition funnel (containing a cotton plug and ca. $10 \mathrm{~g}$ of $4 \AA$ molecular sieves, ${ }^{1}$ and functioning as a Soxhlet extractor) fitted on top with a reflux condenser and a nitrogen inlet adaptor was charged with (S)-(-)- $\alpha, \alpha$-diphenyl-2pyrrolidinemethanol (2.59 g, $10.25 \mathrm{mmol}$, from Aldrich or Lancaster), tri-o-tolylboroxine ${ }^{2}$ (1.21 $\mathrm{g}, 3.42 \mathrm{mmol}$ ) and $40 \mathrm{~mL}$ of toluene. The resulting solution was heated to reflux (bath temperature $\sim 145^{\circ} \mathrm{C}$ ). After $3 \mathrm{~h}$, the reaction mixture was cooled to $c a .60^{\circ} \mathrm{C}$ and the addition funnel and condenser were quickly replaced with a short-path distillation head. The mixture was concentrated by distillation (air-cooling) to a volume of $c a .15 \mathrm{~mL}$. This distillation protocol was repeated three times by re-charging with $3 \times 35 \mathrm{~mL}$ of toluene. The solution was then allowed to cool to room temperature and the distillation head was quickly replaced with a vacuum adaptor. Concentration in vacuo (ca. $0.1 \mathrm{mmHg}, 1 \mathrm{~h})$ afforded the corresponding oxazaborolidine as clear oil. A stock solution $(0.25 \mathrm{M})$ of this catalyst precursor was prepared in either toluene or dichloromethane and stored at $-20^{\circ} \mathrm{C}$. A stock solution $(0.25 \mathrm{M})$ of $(R)-1$ was prepared from $(R)-(+)-\alpha, \alpha$-diphenyl-2-pyrrolidinemethanol in a similar manner.

1 Molecular sieves (pellets) were dried in vacuo at ca. $200{ }^{\circ} \mathrm{C}$ with a gas burner for 10 min prior to use.

2 Corey, E. J.; Shibata, T.; Lee, T. W. J. Am. Chem. Soc. 2002, 124, 3808-3809. 
Oxazaborolidine-Aluminum Bromide Complex $(S)-3$. To an aliquot of the oxazaborolidine precursor $(0.031 \mathrm{mmol}$, theoretical $)$ in dichloromethane $(0.125 \mathrm{~mL})$ at $-40{ }^{\circ} \mathrm{C}$ was added aluminum bromide (1.0 M solution in dibromomethane, $0.025 \mathrm{~mL}, 0.25 \mathrm{mmol})$ dropwise. A strong orange color is immediately observed which immediately faded to a light yellow color. The homogeneous catalyst solution of $(S)-3$ was stirred at $-40{ }^{\circ} \mathrm{C}$ for an additional 30 min. and was ready for the Diels-Alder reaction. The catalyst solution of $(R)-\mathbf{3}$ was prepared from $(R)-\mathbf{1}$ in a similar manner.

${ }^{1} \mathrm{H}$ NMR Analysis of the Oxazaborolidine-AlBr 3 Complex:

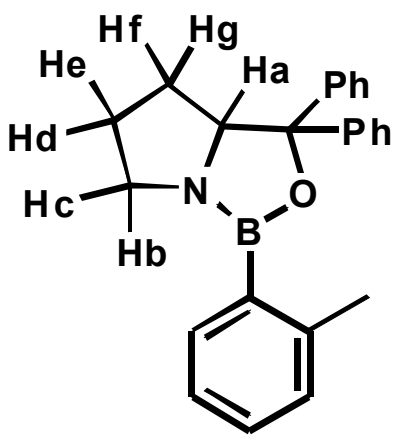

1

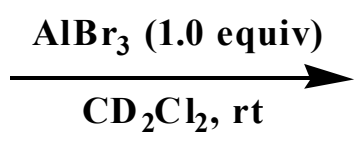

$\mathrm{CD}_{2} \mathrm{Cl}_{2}, \mathrm{rt}$

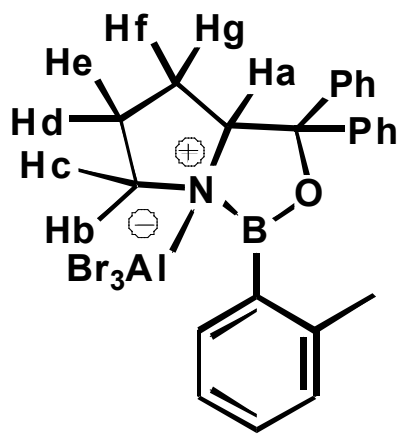

3

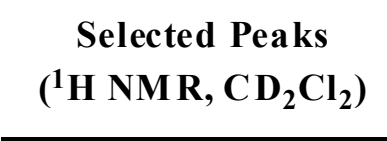

$\mathrm{B}-\mathrm{Ph}-\mathrm{CH}_{3}$

Ha

$\mathrm{Hb}, \mathrm{Hc}$

Hd, He

Hf, Hg
Chemical Shifts (d in ppm)

13

2.63

2.81

4.54

5.26

$3.40,3.24$

$3.51,3.95$

$1.78(2 \mathrm{H})$

$2.15(2 \mathrm{H})$

$1.60,0.95$

$1.92,1.44$

Representative Procedure for the Use of Catalyst 3 for Diels-Alder Reactions. The catalyst solution $(S)-3(0.025 \mathrm{mmol})$ was cooled to $-78^{\circ} \mathrm{C}$ and diethyl fumarate $(0.625 \mathrm{mmol})$ was added, 
followed by cyclopentadiene $(3.13 \mathrm{mmol}, 5$ equiv). The reaction mixture was stirred for the period of time indicated in the tables, quenched with $\mathrm{Et}_{3} \mathrm{~N}$ or $\mathrm{MeOH}(100 \mu \mathrm{L})$, and brought to room temperature. The solvent was removed by rotary evaporation and the crude product was purified by silica gel column chromatography to afford the Diels-Alder adduct.

\section{Physical Data of the Diels-Alder Products:}

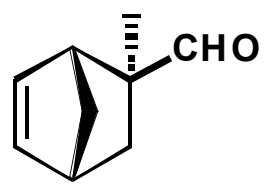

(1R, 2S, 4R)-Bicyclo[2.2.1]hept-5-ene-2-methyl-2-carboxaldehyde (Table 1, entry 1). The physical and spectral data were identical to those previously reported for this compound: ${ }^{1} \mathrm{H}$ NMR $\left(500 \mathrm{MHz}, \mathrm{CDCl}_{3}\right) \delta 9.68(\mathrm{~s}, 1 \mathrm{H}), 6.28(\mathrm{dd}, 1 \mathrm{H}, J=3.1,5.6 \mathrm{~Hz}), 6.09(\mathrm{dd}, 1 \mathrm{H}, J=3.1,5.7 \mathrm{~Hz})$, 2.87 (bs, 1H), 2.80 (bs, 1H), 2.23 (dd, 1H, J = 3.9, $12.0 \mathrm{~Hz}), 1.38(\mathrm{~m}, 2 \mathrm{H}), 1.00$ (s, 3H), 0.75 (bd, $1 \mathrm{H}, J=12.0 \mathrm{~Hz}$ ). Diastereoselectivity (exo-endo ratio, 92:8) was determined by ${ }^{1} \mathrm{H}$ NMR analysis of the crude mixture: $\delta 9.68(\mathrm{~s}, 1 \mathrm{H}$, exo $), 9.38(\mathrm{~s}, 1 \mathrm{H}$, endo). Enantioselectivity $(93 \%$ ee) was determined by reduction with $\mathrm{NaBH}_{4}$ to the corresponding alcohol, conversion to the $(R)$-MTPA ester derivative and ${ }^{1} \mathrm{H}$ NMR integration $\left(500 \mathrm{MHz}, \mathrm{CDCl}_{3}\right): \delta 4.34(\mathrm{~d}, 1 \mathrm{H}$, major), $4.31(\mathrm{~d}, 1 \mathrm{H}$, minor), 4.25 (d, 1H, minor), 4.22 (d, 1H, major).

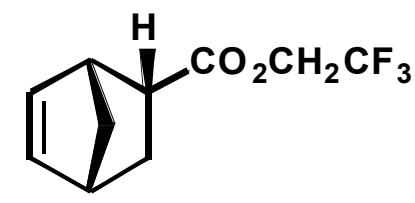

(1R, 2R, 4R)-Bicyclo[2.2.1] hept-5-ene-2-carboxylic Acid 2,2,2-Trifluoroethyl Ester (Table 1, entry 2). Purification by column chromatography (gradient elution with $2-5 \% \mathrm{Et}_{2} \mathrm{O}$-pentane) afforded $135 \mathrm{mg}(98 \%)$ of product as a colorless oil. The physical and spectral data were 
identical to those previously reported for this compound: ${ }^{1} \mathrm{H}$ NMR $\left(400 \mathrm{MHz}, \mathrm{CDCl}_{3}\right) \delta 6.22(\mathrm{dd}$, $1 \mathrm{H}, J=3.2,5.6 \mathrm{~Hz}), 5.92(\mathrm{dd}, 1 \mathrm{H}, J=2.8,5.6 \mathrm{~Hz}), 4.49(\mathrm{dq}, 1 \mathrm{H}, J=8.4,12.8 \mathrm{~Hz}), 4.35(\mathrm{dq}, 1 \mathrm{H}$, $J=8.4,12.8 \mathrm{~Hz}), 3.26(\mathrm{~s}, 1 \mathrm{H}), 3.04(\mathrm{dt}, 1 \mathrm{H}, J=4.0,9.2 \mathrm{~Hz}), 2.94(\mathrm{~s}, 1 \mathrm{H}), 1.95$ (ddd, $1 \mathrm{H}, J=3.6$, 9.2, $11.6 \mathrm{~Hz}), 1.42-1.48(\mathrm{~m}, 2 \mathrm{H}), 1.30(\mathrm{~d}, 1 \mathrm{H}, J=8.0 \mathrm{~Hz}) ;{ }^{13} \mathrm{C} \mathrm{NMR}\left(100 \mathrm{MHz}, \mathrm{CDCl}_{3}\right) \delta$ 173.1, 138.1, 132.0, $123.0(\mathrm{q}, 1 \mathrm{C}, J=276 \mathrm{~Hz}), 60.0(\mathrm{q}, 1 \mathrm{C}, J=36.4 \mathrm{~Hz}), 49.6,45.8,42.9,42.5,29.2$. Diastereoselectivity (endo-exo ratio, 98:2) and enantioselectivity (99\% ee) were determined by GC analysis using a $\mathrm{J} \& \mathrm{~W}$ Scientific Cyclosil-B column $\left(30 \mathrm{~m} \times 0.25 \mathrm{~mm}, 100{ }^{\circ} \mathrm{C}\right)$; retention times: $6.0 \mathrm{~min}($ exo), $6.7 \mathrm{~min}$ (endo, major), $7.5 \mathrm{~min}$ (endo, minor).

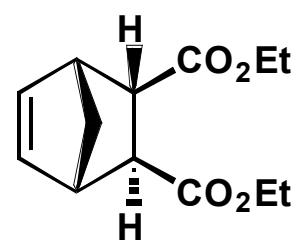

$(1 R, 2 S, 3 S, 4 S)$-Bicyclo[2.2.1]hept-5-ene-2,3-dicarboxylic Acid Diethyl Ester (Table 1, entry 3). Purification by column chromatography (10\% $\mathrm{Et}_{2} \mathrm{O}$-pentane) afforded $142 \mathrm{mg}(95 \%)$ of product as a colorless oil. The physical and spectral data were identical to those previously reported for this compound: ${ }^{1} \mathrm{HNMR}\left(500 \mathrm{MHz}, \mathrm{CDCl}_{3}\right) \delta 6.27(\mathrm{dd}, 1 \mathrm{H}, J=3.3,5.8 \mathrm{~Hz}), 6.06$ (dd, $1 \mathrm{H}, J=2.8,5.3 \mathrm{~Hz}), 4.16$ (q, 2H, $J=7.5 \mathrm{~Hz}$ ), 4.12-4.06 (m, 2H), 3.37 (app. t, 1H, $J=4.0$ Hz), $3.25(\mathrm{~m}, 1 \mathrm{H}), 3.11(\mathrm{~m}, 1 \mathrm{H}), 2.67(\mathrm{dd}, 1 \mathrm{H}, J=1.5,4.5 \mathrm{~Hz}), 1.61(\mathrm{~d}, 1 \mathrm{H}, J=8.5 \mathrm{~Hz}), 1.45-$ $1.43(\mathrm{~m}, 1 \mathrm{H}), 1.27(\mathrm{t}, 3 \mathrm{H}, J=7.5 \mathrm{~Hz}), 1.23(\mathrm{t}, 3 \mathrm{H}, J=7.5 \mathrm{~Hz}) ;{ }^{13} \mathrm{C} \mathrm{NMR}\left(125 \mathrm{MHz}, \mathrm{CDCl}_{3}\right) \delta$ 174.5, 173.3, 137.6, 135.0, 60.8, 60.5, 47.8 (2C), 47.2 (2C), 45.7, 14.2 (2C). Enantioselectivity ( $98 \%$ ee) was determined by HPLC analysis using a $(S, S)$-Whelk-O1 column $(2 \% i$-PrOH in hexanes; $1.0 \mathrm{~mL} / \mathrm{min} ; \lambda 205 \mathrm{~nm}$ ); retention times: $7.4 \min$ (major), $8.5 \mathrm{~min}$ (minor). The 
absolute configuration was determined by comparison of the optical rotation with reported data: $[\alpha]_{\mathrm{D}}^{25}+103\left(c 1.0, \mathrm{CHCl}_{3}, 98 \%\right.$ ee $)$.

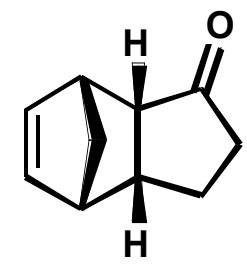

$(1 R, 2 R, 6 R, 7 \mathrm{~S})$-Tricyclo[5.2.1.0 $\left.{ }^{2,6}\right]$ dec-8-en-3-one (Table 1, entry 4). Purification by column chromatography (5\% $\mathrm{Et}_{2} \mathrm{O}$-pentane) afforded $88 \mathrm{mg}(95 \%)$ of product as a colorless oil. The physical and spectral data were identical to those previously reported for this compound: ${ }^{1} \mathrm{H}$ $\operatorname{NMR}\left(400 \mathrm{MHz}, \mathrm{CDCl}_{3}\right) \delta 6.18(\mathrm{dd}, 1 \mathrm{H}, J=2.9,5.9 \mathrm{~Hz}), 6.07$ (dd, 1H, $\left.J=2.9,5.5 \mathrm{~Hz}\right), 3.15$ (bs, 1H), 2.96 (bs, 1H), 2.92 (m, 1H), 2.81 (ddd, 1H, $J=1.1,4.4,9.2 \mathrm{~Hz}), 2.10(\mathrm{~m}, 1 \mathrm{H}), 1.95$ (m, 2H), $1.48(\mathrm{~m}, 2 \mathrm{H}), 1.38(\mathrm{~d}, 1 \mathrm{H}, J=8.8 \mathrm{~Hz}) ;{ }^{13} \mathrm{C} \mathrm{NMR}\left(100 \mathrm{MHz}, \mathrm{CDCl}_{3}\right) \delta$ 222.3, 136.1, 134.7, 54.2, 52.2, 47.4, 46.9, 41.1, 40.5, 22.6. Diastereoselectivity (endo-exo ratio, 97:3) and enantioselectivity ( $92 \%$ ee) were determined by GC analysis using a J \& W Scientific Cyclosil-B column $\left(30 \mathrm{~m} \times 0.25 \mathrm{~mm}, 110{ }^{\circ} \mathrm{C}, 25 \mathrm{psi}\right)$ retention times: $15.4 \mathrm{~min}($ exo), $17.3 \mathrm{~min}$ (endo, minor), $18.4 \min$ (endo, major).

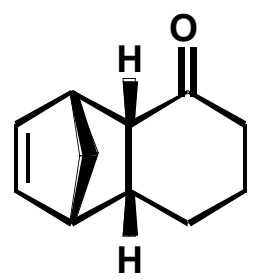

$(1 R, 2 R, 7 R, 8 S)$-Tricyclo[6.2.1.0 $\left.{ }^{2,7}\right]$ undec-9-en-3-one (Table 1, entry 5). Purification by column chromatography ( $5 \% \mathrm{Et}_{2} \mathrm{O}-$ pentane) afforded $100 \mathrm{mg}(99 \%)$ of product as a colorless oil. The physical and spectral data were identical to those previously reported for this compound: ${ }^{1} \mathrm{H}$ 
NMR (400 MHz, $\left.\mathrm{CDCl}_{3}\right) \delta 6.18(\mathrm{dd}, 1 \mathrm{H}, J=2.9,5.9 \mathrm{~Hz}), 6.01(\mathrm{dd}, 1 \mathrm{H}, J=2.9,5.9 \mathrm{~Hz}), 3.27$ (bs, 1H), $2.88(\mathrm{bs}, 1 \mathrm{H}), 2.72(\mathrm{dd}, 1 \mathrm{H}, J=3.7,10.3 \mathrm{~Hz}), 2.67(\mathrm{~m}, 1 \mathrm{H}), 2.32(\mathrm{~m}, 1 \mathrm{H}), 1.94(\mathrm{~m}, 2 \mathrm{H})$, $1.74(\mathrm{~m}, 2 \mathrm{H}), 1.45$ (app. dt, $1 \mathrm{H}, J=1.8,8.4 \mathrm{~Hz}), 1.31$ (d, $1 \mathrm{H}, J=8.4 \mathrm{~Hz}), 0.77(\mathrm{~m}, 1 \mathrm{H}) ;{ }^{13} \mathrm{C}$ NMR (100 MHz, $\left.\mathrm{CDCl}_{3}\right) \delta 215.3,137.5,134.8,51.5,48.2,46.4,45.1,41.3,39.3,27.9,21.7$. Diastereoselectivity (endo-exo ratio, 94:6) was determined by ${ }^{1} \mathrm{H}$ NMR analysis of the crude reaction mixture: $\delta 6.01(\mathrm{dd}, 1 \mathrm{H}, J=2.9,5.9 \mathrm{~Hz}$, endo), $6.09(\mathrm{dd}, 1 \mathrm{H}, J=3.1,5.7 \mathrm{~Hz}$, exo). Enantioselectivity $(95 \%$ ee) was determined by HPLC analysis using a $(S, S)$-Whelk-O1 column ( $1 \% i$-PrOH in hexanes; $1.0 \mathrm{~mL} / \mathrm{min} ; \lambda 205 \mathrm{~nm}$ ); retention times: 10.3 min (major), $11.7 \mathrm{~min}$ (minor).

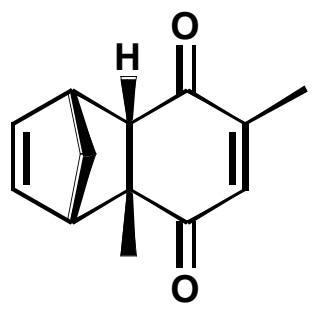

$(1 R, 2 R, 7 S, 8 R)-2,5-D i m e t h y l-t r i c y c l o\left[6.2 .1 .0^{2,7}\right]$ undeca-4,9-diene-3,6-dione (Table 2, entry 1). Purification by column chromatography (gradient elution with $2-10 \%$ EtOAc-hexanes) afforded $125 \mathrm{mg}(99 \%)$ of product as a pale yellow solid. The physical and spectral data were identical to those previously reported for this compound: ${ }^{1} \mathrm{H}$ NMR $\left(500 \mathrm{MHz}, \mathrm{CDCl}_{3}\right) \delta 6.37(\mathrm{~d}$, $1 \mathrm{H}, J=1.5 \mathrm{~Hz}), 6.03(\mathrm{dd}, 1 \mathrm{H}, J=3.0,5.5 \mathrm{~Hz}), 5.88(\mathrm{dd}, 1 \mathrm{H}, J=3.0,5.5 \mathrm{~Hz}), 3.33(\mathrm{~s}, 1 \mathrm{H}), 2.98$ (s, 1H), $2.75(\mathrm{~d}, 1 \mathrm{H}, J=4.0 \mathrm{~Hz}), 1.85(\mathrm{~d}, 3 \mathrm{H}, J=1.5 \mathrm{~Hz}), 1.60(\mathrm{~d}, 1 \mathrm{H}, J=9.5 \mathrm{~Hz}), 1.44(\mathrm{~d}, 1 \mathrm{H}, J$ $=9.0 \mathrm{~Hz}), 1.37(\mathrm{~s}, 3 \mathrm{H}) ;{ }^{13} \mathrm{C} \mathrm{NMR}\left(125 \mathrm{MHz}, \mathrm{CDCl}_{3}\right) \delta 202.6,200.0,150.8,139.1,138.0,134.2$, 56.8, 53.4, 53.0, 49.1, 46.2, 26.2, 16.0. Enantioselectivity (99\% ee) was determined by HPLC analysis using a $(S, S)$-Whelk-O1 column $(1 \% i$-PrOH in hexanes; $0.6 \mathrm{~mL} / \mathrm{min} ; \lambda 254 \mathrm{~nm})$; retention times: 29.7 min (major), 34.9 min (minor). 


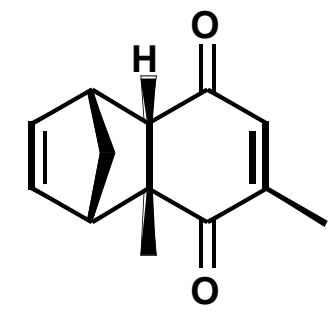

$(1 R, 2 R, 7 S, 8 R)-2,4-D i m e t h y l-t r i c y c l o\left[6.2 .1 .0^{2,7}\right]$ undeca-4,9-diene-3,6-dione (Table 2, entry

2). Purification by column chromatography (gradient elution with $2-10 \%$ EtOAc-hexanes) afforded $125 \mathrm{mg}(99 \%)$ of product as a yellow oil: ${ }^{1} \mathrm{H} \mathrm{NMR}\left(400 \mathrm{MHz}, \mathrm{CDCl}_{3}\right) \delta 6.45(\mathrm{~s}, 1 \mathrm{H})$, $6.04(\mathrm{dd}, 1 \mathrm{H}, J=2.8,5.4 \mathrm{~Hz}), 5.95(\mathrm{dd}, 1 \mathrm{H}, J=2.8,5.8 \mathrm{~Hz}), 3.37$ (s, 1H), $3.04(\mathrm{~s}, 1 \mathrm{H}), 2.78(\mathrm{~d}, 1 \mathrm{H}$, $J=3.6 \mathrm{~Hz}), 1.89(\mathrm{~s}, 3 \mathrm{H}), 1.63(\mathrm{~d}, 1 \mathrm{H}, J=9.2 \mathrm{~Hz}), 1.48(\mathrm{~d}, 1 \mathrm{H}, J=9.2 \mathrm{~Hz}), 1.41(\mathrm{~s}, 3 \mathrm{H}) ;{ }^{13} \mathrm{C}$ NMR (125 MHz, $\left.\mathrm{CDCl}_{3}\right) \delta$ 203.1, 199.3, 151.2, 139.0, 137.7, 134.9, 57.7, 53.6, 52.4, 48.9, 46.3, 26.5, 16.6. IR (film) 2974, $1661,1290 \mathrm{~cm}^{-1}$; LRMS (CI) calcd for $\mathrm{C}_{13} \mathrm{H}_{15} \mathrm{O}_{2}\left(\mathrm{MH}^{+}\right)$203.1, found 203.1; $[\alpha]_{\mathrm{D}}{ }^{25}+106.0\left(c 1.0, \mathrm{CHCl}_{3}, 97 \%\right.$ ee). Enantioselectivity $(97 \%$ ee) was determined by HPLC analysis using a Chiralpak IA column $(1 \% i$-PrOH in hexanes; $1.0 \mathrm{~mL} / \mathrm{min} ; \lambda 254 \mathrm{~nm})$; retention times: $7.8 \mathrm{~min}$ (major), $8.2 \mathrm{~min}$ (minor). ${ }^{3}$

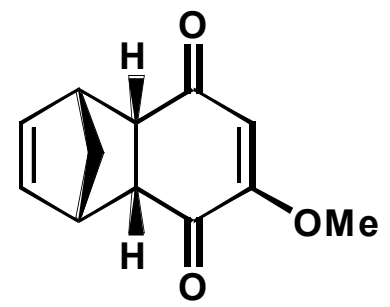

6-Methoxy-4a-methyl-1,4,4a,8a-tetrahydro-1,4-methanonaphthalene-5,8-dione (Table 2, entry 3). Purification by column chromatography (gradient elution with $20-40 \%$ EtOAchexanes) afforded $117 \mathrm{mg}(92 \%)$ of product as a white solid: ${ }^{1} \mathrm{H} \mathrm{NMR}\left(400 \mathrm{MHz}, \mathrm{CDCl}_{3}\right) \delta 6.07$

\footnotetext{
${ }^{3}$ Northcott, C. J.; Valenta, Z. Can. J. Chem. 1987, 65, 1917.
} 
$(\mathrm{dd}, 1 \mathrm{H}, J=2.8,5.6 \mathrm{~Hz}), 6.02(\mathrm{dd}, 1 \mathrm{H}, J=2.8,5.6 \mathrm{~Hz}), 5.87(\mathrm{~s}, 1 \mathrm{H}), 3.70(\mathrm{~s}, 3 \mathrm{H}), 3.52(\mathrm{~d}, 2 \mathrm{H}, J$ $=12.4 \mathrm{~Hz}), 3.25-3.19(\mathrm{~m}, 2 \mathrm{H}), 1.52(\mathrm{~d}, 1 \mathrm{H}, J=8.4 \mathrm{~Hz}), 1.42(\mathrm{~d}, 1 \mathrm{H}, J=8.4 \mathrm{~Hz}) ;{ }^{13} \mathrm{C} \mathrm{NMR}(100$ $\left.\mathrm{MHz}, \mathrm{CDCl}_{3}\right) \delta 198.5,193.8,162.9,135.6,134.6,114.6,56.2,49.1,48.9,48.8,48.3,48.1 ; \mathrm{IR}$ (film) $3005,2950,1692,1645,1599,1228,1159 \mathrm{~cm}^{-1}$; LRMS (CI) calcd for $\mathrm{C}_{12} \mathrm{H}_{13} \mathrm{O}_{3}\left(\mathrm{MH}^{+}\right)$ 204.1, found $204.1 ;[\alpha]_{\mathrm{D}}{ }^{25}+74.3\left(c 1.0, \mathrm{CHCl}_{3}, 72 \%\right.$ ee). Enantioselectivity $(72 \%$ ee) was determined by HPLC analysis using a Chiralpak AD column $(5 \%$ - $\mathrm{PrOH}$ in hexanes; 1.0 $\mathrm{mL} / \mathrm{min} ; \lambda 254 \mathrm{~nm}$ ); retention times: $21.4 \mathrm{~min}$ (major), $23.5 \mathrm{~min}$ (minor).

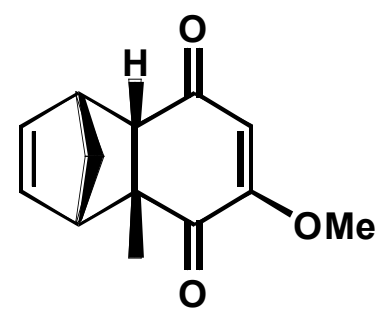

$(1 R, 2 R, 7 S, 8 R)$-4-Methoxy-2-methyl-tricyclo[6.2.1.0 $\left.{ }^{2,7}\right]$ undeca-4,9-diene-3,6-dione (Table 2, entry 4). Purification by column chromatography (gradient elution with 2-10\% EtOAc-hexanes) afforded $135 \mathrm{mg}$ (99\%) of product as a pale white solid: ${ }^{1} \mathrm{H} \mathrm{NMR}\left(400 \mathrm{MHz}, \mathrm{CDCl}_{3}\right) \delta 6.09$ (dd, $1 \mathrm{H}, J=3.2,5.6 \mathrm{~Hz}), 6.00(\mathrm{dd}, 1 \mathrm{H}, J=3.2,5.4 \mathrm{~Hz}), 5.87(\mathrm{~s}, 1 \mathrm{H}), 3.70(\mathrm{~s}, 3 \mathrm{H}), 3.40(\mathrm{~s}, 1 \mathrm{H}), 3.10$ $(\mathrm{s}, 1 \mathrm{H}), 2.82(\mathrm{~d}, 1 \mathrm{H}, J=4.0 \mathrm{~Hz}), 1.69-1.63(\mathrm{~m}, 1 \mathrm{H}), 1.1 .55-1.51(\mathrm{~m}, 1 \mathrm{H}), 1.46(\mathrm{~s}, 3 \mathrm{H}) ;{ }^{13} \mathrm{C}$ NMR $\left(125 \mathrm{MHz}, \mathrm{CDCl}_{3}\right) \delta 198.5,197.6,162.4,137.5,135.1,113.9,57.7,56.4,53.8,52.7,48.7,46.4$, 26.5; IR (film) 2974, 1692, 1653, 1607, 1228, $1066 \mathrm{~cm}^{-1}$; LRMS (CI) calcd for $\mathrm{C}_{13} \mathrm{H}_{15} \mathrm{O}_{3}\left(\mathrm{MH}^{+}\right)$ 219.1, found 219.1; $[\alpha]_{\mathrm{D}}^{25}+107.0\left(c 1.0, \mathrm{CHCl}_{3}, 88 \%\right.$ ee). Enantioselectivity ( $88 \%$ ee) was determined by HPLC analysis using a $(S, S)$-Whelk-O1 column $(3 \% i$-PrOH in hexanes; 1.0 $\mathrm{mL} / \mathrm{min} ; \lambda 254 \mathrm{~nm}$ ); retention times: $51.1 \mathrm{~min}$ (major), $62.0 \mathrm{~min}$ (minor). 


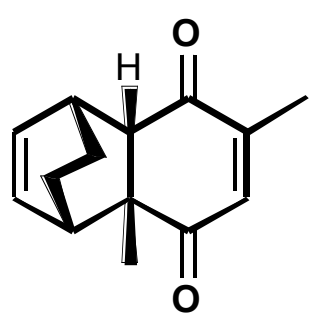

$(1 S, 2 R, 7 S, 8 R)-2,5-D i m e t h y l-t r i c y c l o\left[6.2 .2 .0^{2,7}\right]$ dodeca-4,9-diene-3,6-dione (Table 3, entry

1). Purification by column chromatography (gradient elution with $2-10 \%$ EtOAc-hexanes) afforded $129 \mathrm{mg}(95 \%)$ of product as a pale yellow solid. The physical and spectral data were identical to those previously reported for this compound: ${ }^{1} \mathrm{H}$ NMR $\left(500 \mathrm{MHz}, \mathrm{CDCl}_{3}\right) \delta 6.42(\mathrm{~d}$, $1 \mathrm{H}, J=1.0 \mathrm{~Hz}), 6.19(\mathrm{dd}, 1 \mathrm{H}, J=7.0,7.0 \mathrm{~Hz}), 6.01(\mathrm{dd}, 1 \mathrm{H}, J=7.0,7.0 \mathrm{~Hz}), 2.92-2.93(\mathrm{~m}, 1 \mathrm{H})$, 2.80-2.82 (m, 1H), $2.42(\mathrm{~d}, 1 \mathrm{H}, J=1.5 \mathrm{~Hz}), 1.85(\mathrm{~d}, 3 \mathrm{H}, J=1.5 \mathrm{~Hz}), 1.75-1.81(\mathrm{~m}, 1 \mathrm{H}), 1.62-$ $1.68(\mathrm{~m}, 1 \mathrm{H}), 1.26(\mathrm{tt}, 1 \mathrm{H}, J=3.5,12.0 \mathrm{~Hz}), 1.24(\mathrm{~s}, 3 \mathrm{H}), 1.11(\mathrm{tt}, 1 \mathrm{H}, J=3.5,13.0 \mathrm{~Hz}) ;{ }^{13} \mathrm{C}$ NMR $\left(125 \mathrm{MHz}, \mathrm{CDCl}_{3}\right) \delta$ 202.5, 200.1, 150.0, 138.9, 136.2, 131.7, 58.0, 50.8, 38.9, 36.9, 26.1, 25.8, 18.6, 16.1. Enantioselectivity (99\% ee) was determined by GC analysis using a J \& W Scientific Cyclosil-B column (30 m x $\left.0.25 \mathrm{~mm}, 140{ }^{\circ} \mathrm{C}\right)$; retention times: $29.3 \mathrm{~min}$ (major), 34.9 $\min$ (minor).

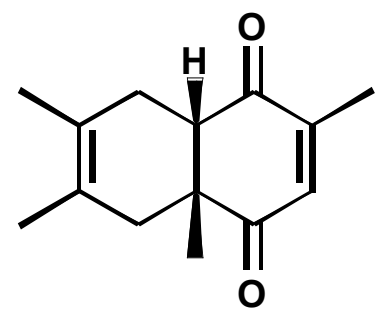

(4aR, 8aS)- 2,4a,6,7-Tetramethyl-4a,5,8,8a-tetrahydro-[1,4]naphthoquinone (Table 3, entry

2). Purification by column chromatography (gradient elution with $2-10 \%$ EtOAc-hexanes) afforded $132 \mathrm{mg}(97 \%)$ of product as a pale yellow solid. The physical and spectral data were identical to those previously reported for this compound: ${ }^{1} \mathrm{H}$ NMR $\left(500 \mathrm{MHz}, \mathrm{CDCl}_{3}\right) \delta 6.39$ - 
$6.40(\mathrm{~m}, 1 \mathrm{H}), 2.77(\mathrm{t}, 1 \mathrm{H}, J=6.0 \mathrm{~Hz}), 2.42-2.47(\mathrm{~m}, 1 \mathrm{H}), 2.33(\mathrm{~d}, 1 \mathrm{H}, J=17.0 \mathrm{~Hz}), 1.99-2.04$ (m, 1H), $1.92(\mathrm{~d}, 3 \mathrm{H}, J=1.0 \mathrm{~Hz}), 1.61(\mathrm{~d}, 1 \mathrm{H}, J=18.0 \mathrm{~Hz}), 1.57(\mathrm{~s}, 3 \mathrm{H}), 1.51(\mathrm{~s}, 3 \mathrm{H}), 1.20(\mathrm{~s}$ $3 \mathrm{H}) ;{ }^{13} \mathrm{C}$ NMR $\left(125 \mathrm{MHz}, \mathrm{CDCl}_{3}\right) \delta 202.5,200.3,148.1,134.8$ (2C), 122.5, 52.6, 48.4, 38.7, 29.3, 22.6, 18.8, 18.5, 16.0. Enantioselectivity (96\% ee) was determined by HPLC analysis using a Chiralpak IA column $(1 \% i$-PrOH in hexanes; $1.0 \mathrm{~mL} / \mathrm{min} ; \lambda 205 \mathrm{~nm})$; retention times: $5.8 \min$ (major), 6.1 min (minor).

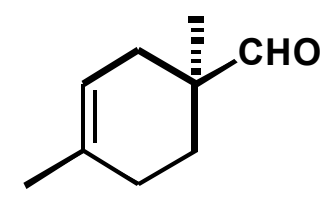

(S)-1,4-Dimethylcyclohex-3-ene-1-carboxaldehyde (Table 3, entry 3). Purification by column chromatography (gradient elution with 2-5\% $\mathrm{Et}_{2} \mathrm{O}$-pentane) afforded $85 \mathrm{mg}$ (99\%) of product as a colorless oil. The physical and spectral data were identical to those previously reported for this compound: ${ }^{1} \mathrm{H}$ NMR $\left(400 \mathrm{MHz}, \mathrm{CDCl}_{3}\right) \delta 9.47(\mathrm{~s}, 1 \mathrm{H}), 5.37$ (bs, 1H), 2.32 (bd, $1 \mathrm{H}, J=17.2 \mathrm{~Hz}), 1.95(\mathrm{~m}, 2 \mathrm{H}), 1.84(\mathrm{~m}, 2 \mathrm{H}), 1.63(\mathrm{~s}, 3 \mathrm{H}), 1.50(\mathrm{~m}, 1 \mathrm{H}), 1.03(\mathrm{~s}, 3 \mathrm{H})$. Enantioselectivity (97\% ee) was determined by HPLC analysis using a Chiralcel OD-H column ( $1 \% i$-PrOH in hexanes; $1.0 \mathrm{~mL} / \mathrm{min} ; \lambda 205 \mathrm{~nm}$ ); retention times: $3.4 \mathrm{~min}$ (minor), $4.1 \mathrm{~min}$ (major).

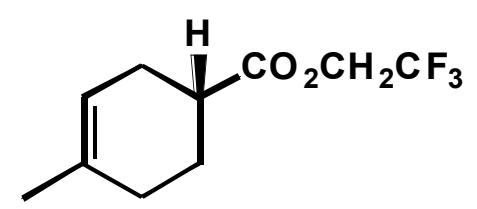

(1R)-4-Methyl-cyclohex-3-enecarboxylic acid 2,2,2-trifluoroethyl ester (Table 3, entry 4). Purification by column chromatography (gradient elution with 2-5\% Et $2 \mathrm{O}$-pentane) afforded 136 mg $(98 \%)$ of product as a colorless oil. The physical and spectral data were identical to those 
previously reported for this compound: ${ }^{1} \mathrm{H}$ NMR $\left(500 \mathrm{MHz}, \mathrm{CDCl}_{3}\right) \delta 5.38-5.37(\mathrm{~m}, 1 \mathrm{H}), 4.52-$ $4.43(\mathrm{~m}, 2 \mathrm{H}), 2.64-2.58(\mathrm{~m}, 1 \mathrm{H}), 2.29-2.23(\mathrm{~m}, 2 \mathrm{H}), 2.07-1.98(\mathrm{~m}, 3 \mathrm{H}), 1.79-1.70(\mathrm{~m}, 1 \mathrm{H}), 1.65$ (s, 3H); ${ }^{13} \mathrm{C}$ NMR $\left(125 \mathrm{MHz}, \mathrm{CDCl}_{3}\right) \delta 174.3,133.8,123.1$ (q, 1C, $\left.J=276 \mathrm{~Hz}\right), 118.7,60.1(\mathrm{q}$, $1 \mathrm{C}, J=36.1 \mathrm{~Hz}), 38.8,29.0,27.4,25.2,23.4$. Enantioselectivity (91\% ee) was determined by $\mathrm{GC}$ analysis using a $\gamma$-TA column $\left(30 \mathrm{~m} \times 0.25 \mathrm{~mm}, 65^{\circ} \mathrm{C}\right)$ retention times: $31.1 \mathrm{~min}$ (minor), 31.9 min (major).

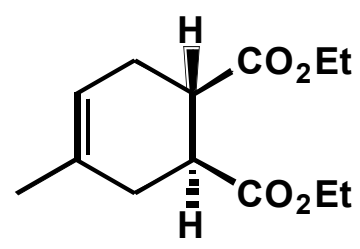

4-Methyl-cyclohex-4-ene-1,2-dicarboxylic acid diethyl ester (Table 3, entry 5). Purification by column chromatography (gradient elution with 2-5\% EtOAc-hexanes) afforded $106 \mathrm{mg}$ (71\%) of product as a colorless oil: ${ }^{1} \mathrm{H}$ NMR $\left(400 \mathrm{MHz}, \mathrm{CDCl}_{3}\right) \delta$ 5.36-5.34 (m, 1H), 4.15-4.07 (m, 4H), 2.86-2.70 (m, 2H), 2.40-2.30 (m, 1H), 2.20-2.28 (m, 1H), 2.03-2.16 (m, 2H), $1.22(\mathrm{t}, 3 \mathrm{H}, J$ $=7.2 \mathrm{~Hz}), 1.21(\mathrm{t}, 3 \mathrm{H}, J=7.2 \mathrm{~Hz}) ;{ }^{13} \mathrm{C} \mathrm{NMR}\left(100 \mathrm{MHz}, \mathrm{CDCl}_{3}\right) \delta$ 174.9, 174.8, 132.1, 118.9, 60.4 (x2), 41.7, 41.1, 32.5, 28.1, 22.9, 14.0; IR (film) 2981, 2935, 1730, 1182, $1035 \mathrm{~cm}^{-1}$; LRMS (CI) calcd for $\mathrm{C}_{13} \mathrm{H}_{21} \mathrm{O}_{4}\left(\mathrm{MH}^{+}\right)$241.1, found 241.1; $[\alpha]_{\mathrm{D}}{ }^{25}+128.0$ (c 1.0, $\mathrm{CHCl}_{3}, 97 \%$ ee). Enantioselectivity $(97 \%$ ee) was determined by HPLC analysis using a $(S, S)$-Whelk-O1 column ( $1 \% i$-PrOH in hexanes; $1.0 \mathrm{~mL} / \mathrm{min} ; \lambda 205 \mathrm{~nm}$ ); retention times: 8.1 min (major), $8.9 \mathrm{~min}$ (minor). 


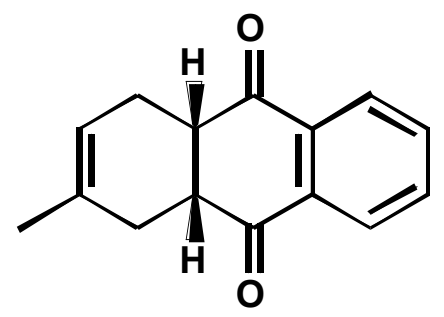

(4aS, 9aR)-1,4,4a,9a-Tetrahydro-2-methyl-anthracene-9,10-dione (Table 3, entry 6). Purification by column chromatography (gradient elution with 5-10\% EtOAc-hexanes) afforded $141 \mathrm{mg}(99 \%)$ of product as a pale yellow oil. The spectral data matched those previously reported for the racemic form of this compound ${ }^{4}:{ }^{1} \mathrm{H}$ NMR $\left(400 \mathrm{MHz}, \mathrm{CDCl}_{3}\right) \delta 8.03(\mathrm{dd}, 2 \mathrm{H}, J$ $=3.2,6.0 \mathrm{~Hz}), 7.73(\mathrm{dd}, 2 \mathrm{H}, J=3.2,6.0 \mathrm{~Hz}), 5.42(\mathrm{~m}, 1 \mathrm{H}), 3.43-3.30(\mathrm{~m}, 2 \mathrm{H}), 2.52-2.41(\mathrm{~m}, 2 \mathrm{H})$,

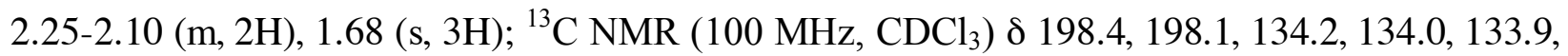
131.8, 126.8 (2C), 118.5, 47.0, 46.4, 28.9, 24.8, 23.4; LRMS (CI) calcd for $\mathrm{C}_{15} \mathrm{H}_{15} \mathrm{O}_{2}\left(\mathrm{MH}^{+}\right)$227.1, found $227.1 ;[\alpha]_{\mathrm{D}}^{25}+20.0\left(c 1.0, \mathrm{CHCl}_{3}, 84 \%\right.$ ee). Enantioselectivity ( $84 \%$ ee) was determined by HPLC analysis using a Chiralpak IA column ( $1 \% i$-PrOH in hexanes; $1.0 \mathrm{~mL} / \mathrm{min} ; \lambda 254 \mathrm{~nm})$; retention times: $10.0 \mathrm{~min}$ (major), $11.2 \mathrm{~min}$ (minor).

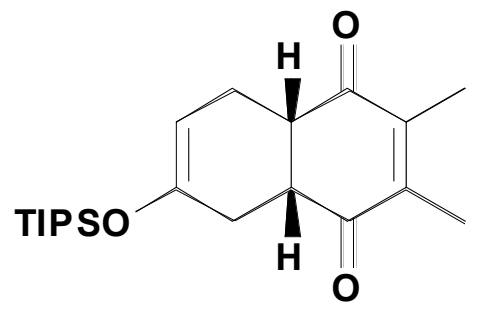

(4aR, 8aS)-2,3-Dimethyl-4a,5,8,8a-tetrahydro-6-(triisopropylsilyl)oxy-naphthalene-1,4dione (Table 3, entry 7). Purification by column chromatography (gradient elution with 2-7\% EtOAc-hexanes) afforded $226 \mathrm{mg}(99 \%)$ of product as a pale yellow oil. The physical and spectral data were identical to those previously reported for this compound: ${ }^{1} \mathrm{H}$ NMR $(500 \mathrm{MHz}$,

\footnotetext{
${ }^{4}$ Fringuelli, F.; Girotti, R.; Pizzo, F.; Vaccaro, L. Org. Lett. 2006, 8, 2487-2489.
} 
$\left.\mathrm{CDCl}_{3}\right) \delta 4.80-4.81(\mathrm{~m}, 1 \mathrm{H}), 3.26(\mathrm{q}, 1 \mathrm{H}, J=5.5 \mathrm{~Hz}), 3.08(\mathrm{q}, 1 \mathrm{H}, J=6.0 \mathrm{~Hz}), 2.50-2.55(\mathrm{~m}$, $1 \mathrm{H}), 2.35-2.41(\mathrm{~m}, 1 \mathrm{H}), 2.15-2.19(\mathrm{~m}, 2 \mathrm{H}), 1.98(\mathrm{~d}, 3 \mathrm{H}, J=1.0 \mathrm{~Hz}), 1.96(\mathrm{~d}, 3 \mathrm{H}, J=1.0 \mathrm{~Hz})$, 1.07-1.16 (m, 3H), 1.05 (d, 18H, $J=6.5 \mathrm{~Hz}) ;{ }^{13} \mathrm{C} \mathrm{NMR}\left(125 \mathrm{MHz}, \mathrm{CDCl}_{3}\right) \delta$ 199.9, 199.0, 148.1, 144.0, 143.7, 100.2, 46.9, 45.9, 28.4, 24.3, 17.9 (6C), 13.0, 12.9, 12.5 (3C). Enantioselectivity (99\% ee) was determined by HPLC analysis using a $(S, S)$-Whelk-O1 column $(1 \% i$-PrOH in hexanes; $1.0 \mathrm{~mL} / \mathrm{min} ; \lambda 254 \mathrm{~nm}$ ); retention times: $8.0 \mathrm{~min}$ (minor), $8.6 \mathrm{~min}$ (major).

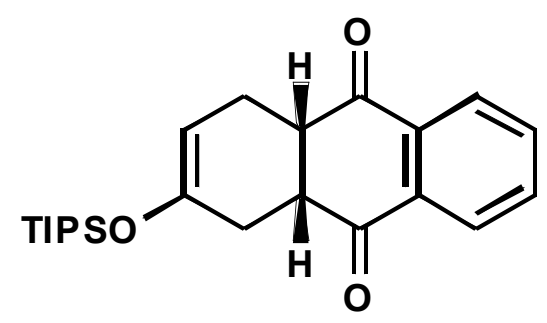

(4aS, 9aR)-1,4,4a,9a-Tetrahydro-2-(triisopropylsilyl)oxy-anthracene-9,10-dione (Table 3, entry 8). The reaction mixture was quenched with $\mathrm{MeOH}$ at $-78^{\circ} \mathrm{C}$ and warmed to rt. The solvent was removed by rotary evaporation and the residue was purified by silica gel chromatography at $-78{ }^{\circ} \mathrm{C}$ to afford $240 \mathrm{mg}(99 \%)$ of the Diels-Alder adduct as a colorless oil. The physical and spectral data were identical to those previously reported for this compound: ${ }^{1} \mathrm{H}$ NMR $\left(500 \mathrm{MHz}, \mathrm{CDCl}_{3}\right) \delta$ 8.03-8.05 (m, 2H), 7.73-7.76 (m, 2H), $4.87(\mathrm{~m}, 1 \mathrm{H}), 3.47$ (dd, 1H, $J$ $=11.0,5.5 \mathrm{~Hz}), 3.29-3.31(\mathrm{~m}, 1 \mathrm{H}), 2.63-2.68(\mathrm{~m}, 1 \mathrm{H}), 2.47-2.53(\mathrm{~m}, 1 \mathrm{H}), 2.25-2.31(\mathrm{~m}, 2 \mathrm{H})$, 1.08-1.27(m, 3H), 1.07 (d, 18H, $J=7.2 \mathrm{~Hz}) ;{ }^{13} \mathrm{C} \mathrm{NMR}\left(125 \mathrm{MHz}, \mathrm{CDCl}_{3}\right) \delta 198.5,197.6,148.5$, 134.6, 134.5, 134.3, 134.1, 127.12, 127.13, 100.5, 47.6, 46.8, 28.7, 24.4, 18.2 (6C), 13.0 (3C). Enantioselectivity $(96 \%$ ee) was determined by HPLC analysis using a Chiralcel OD-H column ( $1 \%$ i-PrOH in hexanes; $1.0 \mathrm{~mL} / \mathrm{min} ; \lambda 254 \mathrm{~nm}$ ); retention times: 5.9 min (major), $6.7 \mathrm{~min}$ (minor). 


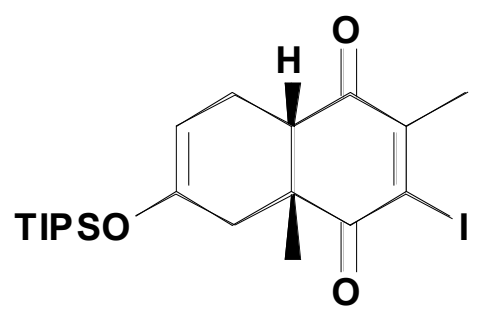

(4aR, 8aS)-3-Iodo-2,4a-dimethyl-4a,5,8,8a-tetrahydro-6-(triisopropylsilyl)oxy-naphthalene1,4-dione (Table ,3 entry 9). Purification by column chromatography (gradient elution with 2\% EtOAc-hexanes) afforded $303 \mathrm{mg}(99 \%)$ of product as a pale yellow oil. The physical and spectral data were identical to those previously reported for this compound: ${ }^{1} \mathrm{H}$ NMR $(500 \mathrm{MHz}$, $\left.\mathrm{CDCl}_{3}\right) \delta 4.76(\mathrm{t}, 1 \mathrm{H}, \mathrm{J}=4.0 \mathrm{~Hz}), 2.74(\mathrm{t}, 1 \mathrm{H}, \mathrm{J}=5.3 \mathrm{~Hz}), 2.55-2.61(\mathrm{~m}, 1 \mathrm{H}), 2.45(\mathrm{dd}, 1 \mathrm{H}, \mathrm{J}=$ 17.5, $2.0 \mathrm{~Hz}), 2.23$ (s, 3H), 2.13-2.20 (m, 1H), 1.82 (d, 1H, J = 17.5 Hz), 1.34(s, 3H), 1.01-1.12 $(\mathrm{m}, 3 \mathrm{H}), 1.00(\mathrm{dd}, 18 \mathrm{H}, \mathrm{J}=7.5,2.0 \mathrm{~Hz}) ;{ }^{13} \mathrm{C} \mathrm{NMR}\left(125 \mathrm{MHz}, \mathrm{CDCl}_{3}\right) \delta$ 194.6, 194.1, 154.9, 147.1, 125.2, 99.1, 51.1, 47.4, 37.0, 23.6 (2C), 22.3, 17.8 (6C), 12.4 (3C). Enantioselectivity (99\% ee) was determined by HPLC analysis using a Chiralcel-OJ column $(1 \%$ i-PrOH in hexanes; $1.0 \mathrm{~mL} / \mathrm{min} ; \lambda 205 \mathrm{~nm}$ ); retention times: $4.3 \mathrm{~min}$ (major), $5.6 \mathrm{~min}$ (minor).

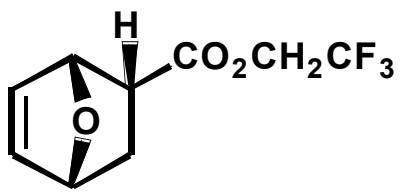

$(1 R, 2 R, 4 R)$-7-Oxa-bicyclo[2.2.1]hept-5-ene-2-carboxylic acid 2,2,2-trifluoroethyl ester (Table 4, entry 1). Purification by column chromatography $\left(\mathrm{CH}_{2} \mathrm{Cl}_{2}\right)$ afforded $137 \mathrm{mg}(99 \%)$ of product as a colorless oil: ${ }^{1} \mathrm{H}$ NMR $\left(500 \mathrm{MHz} \mathrm{CDCl}_{3}\right) \delta 6.46(\mathrm{dd}, 1 \mathrm{H}, J=1.2,5.6 \mathrm{~Hz}), 6.21$ $(\mathrm{dd}, 1 \mathrm{H}, J=1.2,5.6 \mathrm{~Hz}), 5.20(\mathrm{~d}, 1 \mathrm{H}, J=5.5 \mathrm{~Hz}), 5.04(\mathrm{~d}, 1 \mathrm{H}, J=4.5 \mathrm{~Hz}), 4.55-4.47(\mathrm{~m}, 1 \mathrm{H})$, $4.39-4.31(\mathrm{~m}, 1 \mathrm{H}), 3.19(\mathrm{~m}, 1 \mathrm{H}), 2.17-2.11(\mathrm{~m}, 1 \mathrm{H}), 1.61(\mathrm{dd}, 1 \mathrm{H}, J=4.0,11.7 \mathrm{~Hz}) ;{ }^{13} \mathrm{C}$ NMR $\left(100 \mathrm{MHz}, \mathrm{CDCl}_{3}\right): \delta 170.1,137.7,132.5,123.3(\mathrm{q}, 1 \mathrm{C}, J=276.2 \mathrm{~Hz}), 79.3,78.8,60.5(\mathrm{q}, 1 \mathrm{C}, J$ 
$=36.1 \mathrm{~Hz}), 42.7,28.7 . \mathrm{IR}(\mathrm{film}) 3020,2966,2927,1753,1282,1151 \mathrm{~cm}^{-1}$; LRMS (CI) calcd for $\mathrm{C}_{9} \mathrm{H}_{10} \mathrm{~F}_{3} \mathrm{O}_{3}\left(\mathrm{MH}^{+}\right)$223.1, found 223.1; $[\alpha]_{\mathrm{D}}{ }^{25}+68.0$ (c 1.0, $\left.\mathrm{CHCl}_{3}\right)$. Diastereoselectivity (endo:exo ratio, 88:12) was determined by ${ }^{1} \mathrm{H}$ NMR analysis of the crude reaction mixture: $\delta$ $6.46(\mathrm{dd}, 1 \mathrm{H}, J=1.5,6.0 \mathrm{~Hz}$, endo), $6.42(\mathrm{dd}, 1 \mathrm{H}, J=1.5,5.8 \mathrm{~Hz}$, exo $)$. Enantioselectivity (99\% ee) was determined by HPLC analysis using a Chiralpak-IA column $(2 \% i$-PrOH in hexanes; 0.6 $\mathrm{mL} / \mathrm{min} ; \lambda 205 \mathrm{~nm}$ ); retention times: $12.2 \mathrm{~min}$ (endo-minor), $12.6 \mathrm{~min}$ (endo-major), 14.7 (exominor), 15.6 (exo-major). The absolute configuration was determined by comparison of sign of the optical rotation with reported data after converting to the corresponding carboxylic acid. ${ }^{5}$

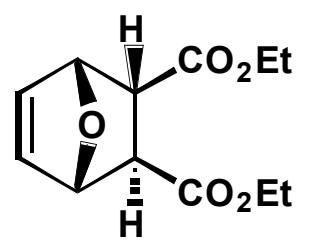

$(1 R, 2 R, 3 R, 4 S)$-7-Oxa-bicyclo[2.2.1] hept-5-ene-2,3-dicarboxylic acid diethyl ester (Table 4, entry 2). Purification by column chromatography $\left(\mathrm{CH}_{2} \mathrm{Cl}_{2}\right)$ afforded $105 \mathrm{mg}(70 \%)$ of product as a pale yellow oil: ${ }^{1} \mathrm{H}$ NMR $\left(400 \mathrm{MHz}, \mathrm{CDCl}_{3}\right) \delta 6.49(\mathrm{dd}, 1 \mathrm{H}, J=1.2,6.0 \mathrm{~Hz}), 6.31(\mathrm{dd}, 1 \mathrm{H}$, $J=1.2,6.0 \mathrm{~Hz}), 5.22-5.18(\mathrm{~m}, 2 \mathrm{H}), 4.22-4.02(\mathrm{~m}, 4 \mathrm{H}), 3.58(\mathrm{t}, 1 \mathrm{H}, J=4.4 \mathrm{~Hz}), 2.81(\mathrm{~d}, 1 \mathrm{H}, J=$ $3.6 \mathrm{~Hz}), 1.26(\mathrm{t}, 3 \mathrm{H}, J=7.2 \mathrm{~Hz}), 1.21(\mathrm{t}, 3 \mathrm{H}, J=7.6 \mathrm{~Hz}) ;{ }^{13} \mathrm{C} \mathrm{NMR}\left(100 \mathrm{MHz}, \mathrm{CDCl}_{3}\right) \delta 171.9$, 170.8, 136.7, 134.8, 82.5, 79.1, 61.2, 60.9, 47.4 (2C), 14.0; IR (film) 3020, 2974, 1761, 1282, $1151 \mathrm{~cm}^{-1}$; LRMS (CI) calcd for $\mathrm{C}_{12} \mathrm{H}_{17} \mathrm{O}_{5}\left(\mathrm{MH}^{+}\right)$241.1, found 241.1; $[\alpha]_{\mathrm{D}}^{25}+43.5(c$ 1.0, $\mathrm{CHCl}_{3}, 99 \%$ ee). Enantioselectivity (99\% ee) was determined by HPLC analysis using a Chiralpak-IA ( $5 \% i$-PrOH in hexanes; $1.0 \mathrm{~mL} / \mathrm{min} ; \lambda 205 \mathrm{~nm}$ ); retention times: $8.3 \mathrm{~min}$ (major), $10.3 \min$ (minor).

${ }^{5}$ Ogawa, S.; Iwasawa, Y.; Suami, T. Chem. Lett. 1984, 355-356. 


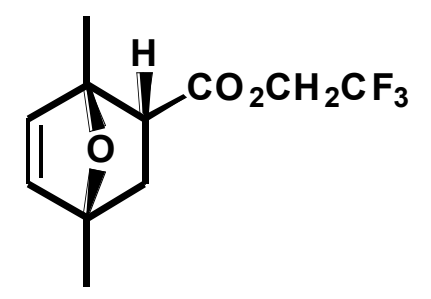

$(1 R, \quad 2 R, \quad 4 R)-1,4-D i m e t h y l-7-o x a-b i c y c l o[2.2 .1] h e p t-5-e n e-2-c a r b o x y l i c \quad$ acid $\quad 2,2,2-$ trifluoroethyl ester (Table 4, entry 3). Purification by column chromatography (10\% EtOAchexanes) afforded $154 \mathrm{mg}(99 \%)$ of product as a colorless oil: ${ }^{1} \mathrm{H}$ NMR $\left(400 \mathrm{MHz}, \mathrm{CDCl}_{3}\right) \delta$ $6.26(\mathrm{~d}, 1 \mathrm{H}, J=5.6 \mathrm{~Hz}), 6.04(\mathrm{~d}, 1 \mathrm{H}, J=5.6 \mathrm{~Hz}), 4.61-4.50(\mathrm{~m}, 1 \mathrm{H}), 4.36-4.26(\mathrm{~m}, 1 \mathrm{H}), 2.99(\mathrm{dd}$, $1 \mathrm{H}, J=3.6,9.2 \mathrm{~Hz}), 2.03-1.98(\mathrm{~m}, 1 \mathrm{H}), 1.89-1.84(\mathrm{~m}, 1 \mathrm{H}), 1.74(\mathrm{~s}, 3 \mathrm{H}), 1.59(\mathrm{~s}, 3 \mathrm{H}) ;{ }^{13} \mathrm{C} \mathrm{NMR}$ $\left(100 \mathrm{MHz}, \mathrm{CDCl}_{3}\right) \delta 170.7,140.5,135.9,122.9(\mathrm{q}, 1 \mathrm{C}, J=276.1 \mathrm{~Hz}), 87.3,86.2,60.1(\mathrm{q}, 1 \mathrm{C}, J$ $=36.4 \mathrm{~Hz}$ ), 50.8, 38.1, 18.5, 18.4; IR (film) 2981, 2935, 1753, 1684, 1282, $1166 \mathrm{~cm}^{-1}$; LRMS (CI) calcd for $\mathrm{C}_{11} \mathrm{H}_{14} \mathrm{~F}_{3} \mathrm{O}_{3}\left(\mathrm{MH}^{+}\right)$251.1, found 251.1; $[\alpha]_{\mathrm{D}}^{25}+34.0$ (c 1.0, $\mathrm{CHCl}_{3}$, pure endo, 94\% ee). Diastereoselectivity (endo:exo ratio, 90:10) was determined by ${ }^{1} \mathrm{H}$ NMR analysis of the crude reaction mixture: $\delta 6.17(\mathrm{~d}, 1 \mathrm{H}, J=5.6 \mathrm{~Hz}$, exo $), 6.04(\mathrm{~d}, 1 \mathrm{H}, J=5.6 \mathrm{~Hz}$, endo). Enantioselectivity (94\% ee) was determined by HPLC analysis using a Chiralcel-OJ column (1\% $i$-PrOH in hexanes; $1.0 \mathrm{~mL} / \mathrm{min} ; \lambda 205 \mathrm{~nm}$ ); retention times: $5.3 \mathrm{~min}$ (major), $5.6 \mathrm{~min}$ (minor).

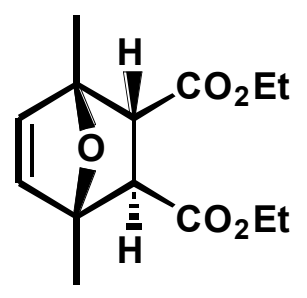

$(1 R, 2 R, 3 R, 4 S)$-1,4-Dimethyl-7-oxa-bicyclo[2.2.1]hept-5-ene-2,3-dicarboxylic acid diethyl ester (Table 4, entry 4). The reaction was quenched with $100 \mu \mathrm{L}$ of saturated $\mathrm{NaHCO}_{3}$ (aq.) at 
$-40{ }^{\circ} \mathrm{C}$. After warming to $\mathrm{rt}, 5 \mathrm{~mL}$ of water was added and the mixture was extracted with ether $(10 \mathrm{~mL} \times 2)$. The combined organic layers were washed with brine once, dried over $\mathrm{Na}_{2} \mathrm{SO}_{4}$, and concentrated to afford the crude product as a pale yellow oil: ${ }^{1} \mathrm{H}$ NMR $\left(300 \mathrm{MHz}, \mathrm{CDCl}_{3}\right) \delta$ $6.24(\mathrm{~d}, 1 \mathrm{H}, J=5.4 \mathrm{~Hz}), 6.15(\mathrm{~d}, 1 \mathrm{H}, J=5.4 \mathrm{~Hz}), 4.28-4.02(\mathrm{~m}, 4 \mathrm{H}), 3.31(\mathrm{~d}, 1 \mathrm{H}, J=4.5 \mathrm{~Hz})$, $2.87(\mathrm{~d}, 1 \mathrm{H}, J=4.5 \mathrm{~Hz}), 1.75(\mathrm{~s}, 3 \mathrm{H}), 1.50(\mathrm{~s}, 3 \mathrm{H}), 1.27(\mathrm{t}, 3 \mathrm{H}, J=7.2 \mathrm{~Hz}), 1.21(\mathrm{t}, 3 \mathrm{H}, J=7.2$ $\mathrm{Hz}) ;{ }^{13} \mathrm{C} \mathrm{NMR}\left(125 \mathrm{MHz}, \mathrm{CDCl}_{3}\right) \delta 171.9,171.1,140.3,138.4,88.4,87.2,60.9,60.7,55.1,53.7$, $18.1,16.2,14.2,14.1$. The reaction conversion of $72 \%$ was determined by ${ }^{1} \mathrm{H}$ NMR of the crude product. Due to the tendency of the product to gradually decompose via possibly a retro-DielsAlder pathway, it was immediately hydrogenated with Pd black in THF at rt under 1 atm of $\mathrm{H}_{2}$ to afford the hydrogenated derivative.

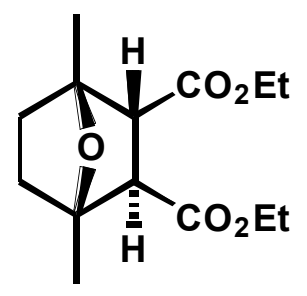

$(1 R, 2 R, 3 R, 4 S)$-1,4-Dimethyl-7-oxa-bicyclo[2.2.1]heptane-2,3-dicarboxylic acid diethyl ester. Purification by column chromatography (10\% EtOAc-hexanes) afforded $121 \mathrm{mg} \mathrm{(72 \% )} \mathrm{of}$ the hydrogenated derivative as a colorless oil: ${ }^{1} \mathrm{H}$ NMR (400 MHz, $\left.\mathrm{CDCl}_{3}\right) \delta$ 4.21-4.08 (m, 4H), $3.29(\mathrm{dd}, 1 \mathrm{H}, J=2.0,5.2 \mathrm{~Hz}), 3.29(\mathrm{~d}, 1 \mathrm{H}, J=5.2 \mathrm{~Hz}), 1.85-1.60(\mathrm{~m}, 3 \mathrm{H}), 1.62(\mathrm{~s}, 3 \mathrm{H}), 1.58-$ $1.50(\mathrm{~m}, 1 \mathrm{H}), 1.40(\mathrm{~s}, 3 \mathrm{H}), 1.26(\mathrm{t}, 3 \mathrm{H}, J=7.2 \mathrm{~Hz}), 1.25(\mathrm{t}, 3 \mathrm{H}, J=7.2 \mathrm{~Hz}) ;{ }^{13} \mathrm{C} \mathrm{NMR}(100 \mathrm{MHz}$, $\left.\mathrm{CDCl}_{3}\right) \delta 172.5,171.4,86.2,85.2,60.8(2 \mathrm{C}), 57.0,55.2,38.8,33.4,20.6,18.9,14.2(2 \mathrm{C}) ; \mathrm{IR}$ (film) 2981, 1730, 1166, $1035 \mathrm{~cm}^{-1}$; LRMS (CI) calcd for $\mathrm{C}_{14} \mathrm{H}_{23} \mathrm{O}_{5}\left(\mathrm{MH}^{+}\right)$271.1, found 271.1; $[\alpha]_{\mathrm{D}}^{25}+26.1\left(c 1.0, \mathrm{CHCl}_{3}, 91 \%\right.$ ee). Enantioselectivity (91\% ee) was determined by HPLC 
analysis using a Chiralcel OD-H column $(2 \% i-\mathrm{PrOH}$ in hexanes; $1.0 \mathrm{~mL} / \mathrm{min} ; \lambda 230 \mathrm{~nm})$; retention times: 5.4 min (major), 6.4 min (minor).

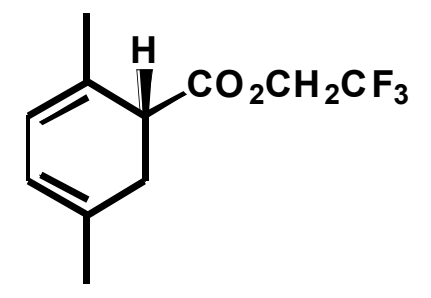

(1R)-2,5-Dimethylcyclohexa-2,4-diene-1-carboxylic acid 2,2,2-trifluoroethyl ester. To a mixture of $(1 R, 2 R, 4 R)$-1,4-dimethyl-7-oxa-bicyclo[2.2.1]hept-5-ene-2-carboxylic acid 2,2,2trifluoroethyl ester ( $151 \mathrm{mg}, 0.603 \mathrm{mmol}$, pure endo) and excess activated $\mathrm{Zn}$ dust in $1.0 \mathrm{~mL}$ of $\mathrm{CH}_{3} \mathrm{CN}$ was added slowly $\mathrm{TMSBr}\left(1.81 \mathrm{mmol}, 239 \mu \mathrm{L}, 3.0\right.$ equiv) at $0{ }^{\circ} \mathrm{C}$. The resulting heterogeneous mixture was stirred at $0{ }^{\circ} \mathrm{C}$ for $1 \mathrm{~h}$ and quenched with water. The mixture was filtered, extracted with pentane, dried over $\mathrm{Na}_{2} \mathrm{SO}_{4}$ and concentrated to give the crude product. Purification by column chromatography (gradient elution with 1-3\% Et $2 \mathrm{O}-$ pentane) afforded 107 $\mathrm{mg}(76 \%)$ of the desired product as a colorless oil: ${ }^{1} \mathrm{H}$ NMR $\left(400 \mathrm{MHz}, \mathrm{CDCl}_{3}\right) \delta 5.78(\mathrm{~m}, 1 \mathrm{H})$, $5.61(\mathrm{~m}, 1 \mathrm{H}), 4.56-4.40(\mathrm{~m}, 2 \mathrm{H}), 3.12(\mathrm{dd}, 1 \mathrm{H}, J=5.6,8.4 \mathrm{~Hz}), 2.57-2.37(\mathrm{~m}, 2 \mathrm{H}), 1.88(\mathrm{~d}, 3 \mathrm{H}, J$ $=0.8 \mathrm{~Hz}), 1.80(\mathrm{~s}, 3 \mathrm{H}) ;{ }^{13} \mathrm{C} \mathrm{NMR}\left(100 \mathrm{MHz}, \mathrm{CDCl}_{3}\right) \delta 172.2,132.2,127.2,123.0(\mathrm{q}, 1 \mathrm{C}, J=$ $275.8 \mathrm{~Hz}$ ), 122.8, 118.9, 60.3 (q, 1C, $J=36.6 \mathrm{~Hz}$ ), 43.9, 31.2, 22.7, 21.9; IR (film) 2974, 2920, 1753, 1282, $1159 \mathrm{~cm}^{-1}$; LRMS (CI) calcd for $\mathrm{C}_{11} \mathrm{H}_{14} \mathrm{~F}_{3} \mathrm{O}_{2}\left(\mathrm{MH}^{+}\right)$235.1, found 235.1; $[\alpha]_{\mathrm{D}}{ }^{25}$ +198.5 (c 1.0, $\mathrm{CHCl}_{3}, 93 \%$ ee). Enantioselectivity $(93 \%$ ee) was determined by $\mathrm{GC}$ analysis using a J \& W Scientific Cyclosil-B column $\left(30 \mathrm{~m}\right.$ x $\left.0.25 \mathrm{~mm}, 100{ }^{\circ} \mathrm{C}\right)$; retention times: 15.1 $\min$ (major), $16.8 \min$ (minor). 